\title{
Tratamento não operatório de lesão renal e esplênica: relato de caso
}

\section{Non-operative treatment of renal and splenic injury: case report}

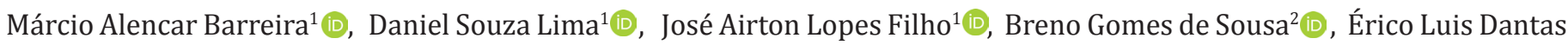 \\ Diógenes Saldanha ${ }^{3}$ (D), Paulo Roberto Montezuma Sales ${ }^{3}$ (D) \\ 1. Cirurgião Geral do Hospital Instituto Dr. José Frota (IJF), Fortaleza, CE, Brasil. 2. Residente de Cirurgia Geral do Hospital Instituto Dr. José Frota (IJF), \\ Fortaleza, CE, Brasil. 3. Urologista do Hospital Instituto Dr. José Frota (IJF), Fortaleza, CE, Brasil.
}

\section{Resumo}

Introdução: em algumas situações, o trauma abdominal contuso com lesão renal ou esplênica pode ser conduzido com tratamento não operatório (TNO). Uma lesão simultânea de rim e baço não apresenta uma conduta padrão e existem situações limítrofes que necessitam de maior discussão. Relato de caso: paciente com lesão renal complexa e hemoperitônio secundário a lesão esplênica que teve boa evolução após compensação clínica do sangramento. Conclusão: o TNO de lesões combinadas do rim e do baço pode ser possível em algumas situações.

Palavras-chave: Ferimentos e lesões. Ferimentos não penetrantes. Traumatismos abdominais

\begin{abstract}
Introduction: In some situations, blunt abdominal trauma with renal or splenic injury can be conducted with non-operative treatment (NOT). A simultaneous lesion of the kidney and spleen does not present a standard conduct and there are borderline situations that need further discussion. Case report: patient with complex renal injury and hemoperitoneum secondary to splenic lesion who had a good evolution after clinical bleeding compensation. Conclusion: NOT of combined kidney and spleen lesions may be possible in some situations.
\end{abstract}

Key words: Wounds and Injuries. Wounds, nonpenetrating. Abdominal injuries.

\section{INTRODUÇÃO}

No trauma abdominal fechado, os órgãos mais frequentemente lesados são o fígado e o baço ${ }^{1}$. O trauma urológico mais frequente é o renal que ocorre predominantemente em adultosjovens, do sexo masculino e a etiologia é predominantemente contundente devido a acidentes com veículos motorizados ${ }^{2}$. Menos frequentemente, os rins podem apresentar lesões traumáticas devido às lesões penetrantes ou ao trauma por desaceleração ${ }^{3}$.

Os rins são órgãos retroperitoneais, protegidos por uma camada de gordura perirrenal e contidos pela fáscia de Gerota. A sua anatomia contribui para a sua proteção. A hematúria está presente em até $90 \%$ dos pacientes com trauma renal, porém a sua intensidade não se correlaciona diretamente com o grau de lesão renal ${ }^{4}$.

Uma maior disponibilidade de exames de imagem, possibilidade de utilizar procedimentos cirúrgicos minimamente invasivos e evolução nos cuidados intensivos levaram a uma melhor aceitação do tratamento não operatório (TNO) das lesões renais, por trauma fechado, que se mostrou seguro e eficaz ${ }^{4}$. No entanto, uma lesão simultânea de rim e baço não apresenta uma conduta-padrão e existem situações limítrofes que necessitam de maior discussão.

\section{RELATO DO CASO}

Homem, 17 anos, veio encaminhado para o Hospital Instituto Dr. José Frota (IJF) com história de trauma abdominal fechado devido à queda de moto, há 30 horas, apresentando dor abdominal e hipotensão arterial sistêmica que foi revertida, apenas, com hidratação vigorosa. Não apresentava hematúria.

$\mathrm{Na}$ sala de reanimação do IJF, o paciente se encontrava consciente e orientado (Escala de Coma de Glasgow (ECG) de 15) apresentando sinais vitais de estabilidade hemodinâmica (Pressão arterial de 128x54 mmHg, frequência cardíaca (FC) de 90 bpm e frequência respiratória (FR) de 24 irpm). 0 abdômen estava distendido e doloroso à palpação superficial do flanco esquerdo.

Foi realizado um FAST abdominal que mostrou grande quantidade de liquido abdominal e foram colhidos exames laboratoriais que evidenciaram uma queda importante da hemoglobina $(\mathrm{Hb})$ para $7,1 \mathrm{~g} / \mathrm{dL}$. Foi iniciada a transfusão de 2 concentrados de hemácias $(\mathrm{CH})$. Em seguida, o paciente foi encaminhado para realizar uma tomografia computadorizada (TC) com contraste do abdômen e radiografia de tórax e bacia. As radiografias não evidenciaram alterações e a tomografia de abdômen (Fig. 1 e 2) mostrou as alterações abaixo. 
Figura 1. Corte coronal, na fase arterial, mostrando uma lesão esplênica grau III associado a grande quantidade de liquido livre intra-abdominal e rim esquerdo com importante laceração e extravasamento de contraste (grau IV). Presença de extenso hematoma retroperitoneal deslocando o rim anteriormente na cavidade abdominal.

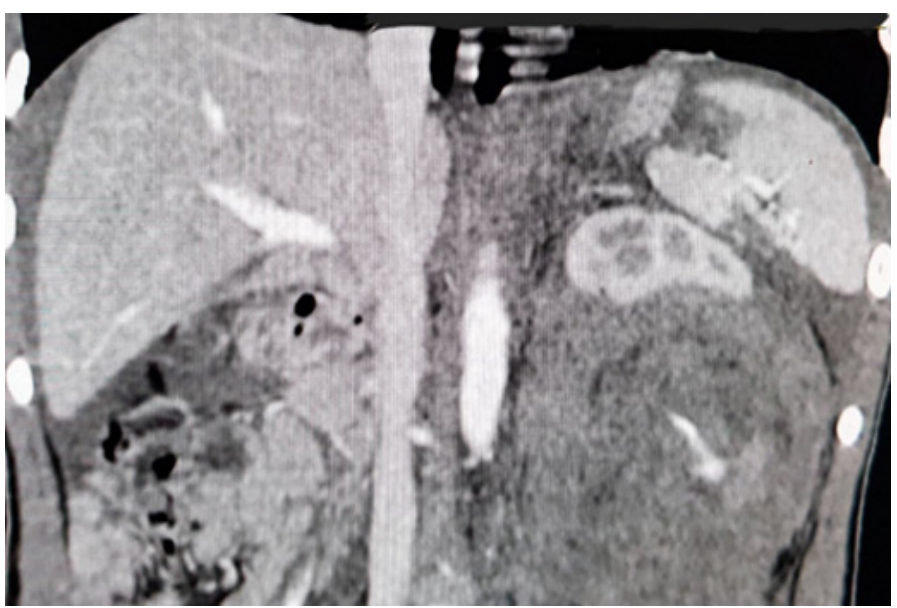

Figura 2. Corte sargital evidenciando o extenso hematoma perirenal esquerdo $(17 \times 10 \mathrm{~cm})$ associado à lesão renal esquerda com extravasamento de contraste e ausência de caracterização de opacificação do ureter ipsilateral. Liquido livre na cavidade abdominal mais evidente acima do rim direito.

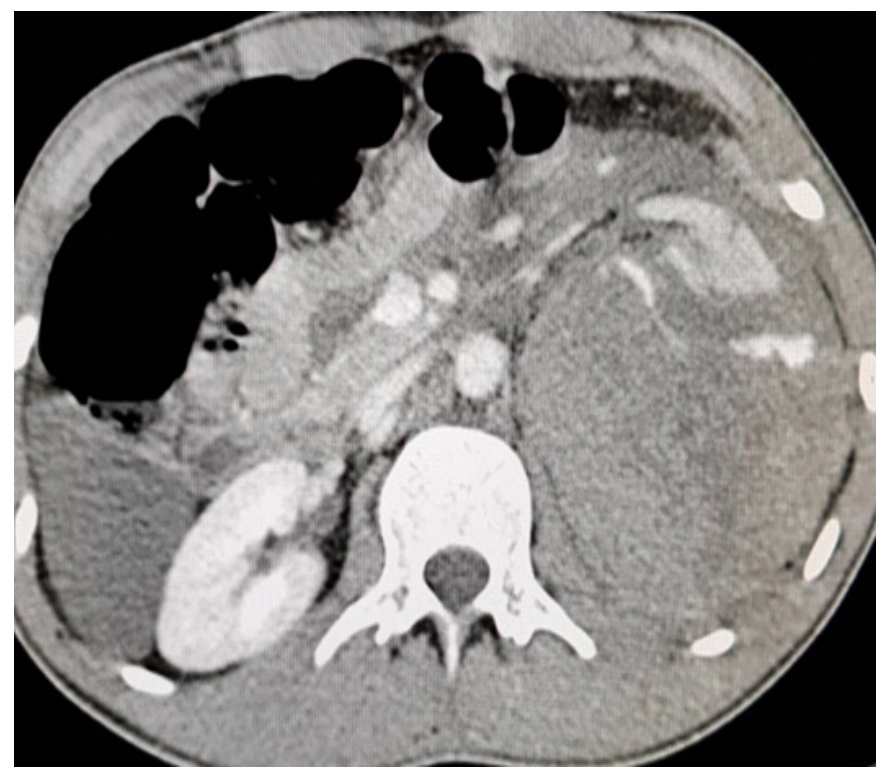

Optou-se por monitorização contínua do paciente, acompanhamento laboratorial e reavaliações a cada 3 horas pela equipe de clínicos, cirurgiões e urologistas presentes no plantão. A Hb, após a transfusão, foi de $7,7 \mathrm{~g} / \mathrm{dL}$, e o paciente permaneceu estável hemodinamicamente. No terceiro dia de internamento hospitalar, (DIH) evoluiu com melhora da dor abdominal e fome, sendo iniciada a dieta e repetida a TC de abdômen que evidenciou hematoma perirenal à esquerda medindo $18,4 \times 9,3 \times 8,8 \mathrm{~cm}$ com volume estimado de 780 $\mathrm{ml}$, rechaçando o rim anteriormente e estável em relação ao exame anterior. Presença de lacerações no $1 \backslash 3$ médio e inferior do rim esquerdo. Não houve extravasamento de contraste na fase intravascular ou na fase excretora, com excreção adequada através do sistema coletor até a bexiga. Observaram-se, ainda, pequenas lacerações esplênicas e acentuada quantidade de hemoperitôneo. A Hb voltou a cair $(6,6 \mathrm{~g} / \mathrm{dL})$, sendo indicada a transfusão de mais $2 \mathrm{CH}$ que elevaram a $\mathrm{Hb}$ para 7,9. Nos dias seguintes, o paciente permaneceu estável hemodinamicamente e foi transferido para a enfermaria no $6 \mathrm{DIH}$. No $15 \mathrm{DIH}$ foi realizada uma USG que mostrou rim esquerdo com evidências de lesão prévia da cápsula, parênquima e sistema coletor do rim esquerdo, apresentando hematoma contido ao seu redor, medindo $12 \times 9,6 \times 4,9 \mathrm{~cm}$. Ausência de líquido livre em cavidade abdominal. Evoluiu bem na enfermaria e recebeu alta no 17 DIH com Hb de 10,9 g/dL, após reposição de ferro oral. A função renal não apresentou alterações em nenhum momento, mesmo com a realização de 2 exames contrastados.

O paciente retornou para o ambulatório de Cirurgia Geral, após três meses do acidente, sem queixas. USG de abdômen e função renal normais. Assim, recebeu alta ambulatorial.

\section{DISCUSSÃO}

O TNO é utilizado em mais de $90 \%$ dos casos de trauma renal fechado, com taxa de nefrectomia de 5,4\%. Porém, essa taxa aumenta quando se avaliam apenas pacientes com lesão complexas ${ }^{2}$. Existe um aumento no TNO do trauma renal que apresenta uma taxa maior em hospitais com alto volume de casos. Logo, a centralização do atendimento de pacientes com trauma renal em hospitais terciários com alto volume desse tipo de trauma e urologistas de plantão pode ser uma estratégia para melhorar as taxas de salvamento renal ${ }^{5}$. O TNO não é recomendado se não existir uma equipe de cirurgiões disposta a acompanhar o paciente de forma seriada, tendo acesso rápido a exames de imagem e com disponibilidade para realizar uma laparotomia de emergência nas 24 horas do dia ${ }^{6}$.

A conduta conservadora é associada à menor morbidade e ao menor tem $\neg$ po de internamento, evitando as complicações das cirurgias, per-da do órgão envolvido, possibilitando um retorno mais precoce às atividades habituais. Na grande maioria dos casos, os óbitos ocorrem devido a outras lesões associadas e não ao trauma renal ${ }^{7}$ e a asso-ciação com outras lesões abdominais aumenta a chance de tratamento cirúrgico ${ }^{8}$.

O TNO de lesões contusas de baço para pacientes admitidos em hospitais de referência para atendimento ao trauma é seguro, seguindo-se rígido protocolo de seleção e tratamento ${ }^{9}$. O grau da lesão esplênica se tornou um dos fatores mais importantes para essa tomada de decisão e um importante fator preditor para falhas no $\mathrm{TNO}^{10}$. Neste caso, o trauma esplênico foi de baixa gravidade, contribuindo para a tomada de decisão conservadora. No entanto, a literatura mostra que, até lesões esplênicas complexas, podem ser acompanhadas de forma segura ${ }^{9}$. 
Uma análise de 24 casos com trauma abdominal contuso com lesão renal de alto grau (IV ou V) mostrou que 2 pacientes precisaram realizar uma nefrectomia por instabilidade hemodinâmica e tinham lesão esplênica grave associada. Complicações do TNO ocorreram em 5 pacientes e foram resolvidas, na maioria dos casos, com medidas clinicas ${ }^{11}$.

Um estudo selecionou 26 pacientes com trauma abdominal contuso e lesão esplênica para TNO. A média do escore revisado do trauma (ERT) foi 7,66 $\pm 0,499$. A pontuação máxima do ERT é de 8 e leva em consideração a ECG, PAS e FR. É uma escala efetiva para avaliar a mortalidade geral no trauma e o valor acima de sete está relacionado a uma alta probabilidade de sobrevida ${ }^{12}$.

Dez pacientes $(38,5 \%)$ necessitaram de transfusão de sangue, com uma média de 1,92 $\pm 1,77 \mathrm{CH}$ por paciente. Apenas, dois pacientes $(7,7 \%)$ foram operados durante o acompanhamento. Assim, a necessidade de transfusão sanguínea não foi um impedimento para o TNO dos traumas abdominais contusos ${ }^{9}$.

O paciente do relato de caso tinha um ERT de 8 e necessitou de $4 \mathrm{CH}$, mas, em nenhum momento, apresentou hipotensão artéria sistêmica. Outros fatores que contribuíram para o TNO foram o tempo entre o acidente e a admissão no hospital (36 horas), o fato de ser jovem e a estabilização do sangramento renal evidenciado na segunda TC.

Para os pacientes com trauma de órgãos abdominais sólidos, a estabilidade hemodinâmica é importante para definir a conduta. Pacientes estáveis podem ser encaminhados para realizar uma tomografia computadorizada (TC) com contraste. ${ }^{13}$

Deve-se considerar a possibilidade de uma intervenção precoce em pacientes hemodinamicamente estáveis, mas, com alto risco de perda contínua de sangue quando existe uma lesão penetrante e a presença de alguns achados na TC (extravasamento do contraste intravascular, laceração medial e hematoma perirrenal com diâmetro $>3,5 \mathrm{~cm})^{14}$.

Procedimentos cirúrgicos minimamente invasivos podem ser utilizados para evitar uma cirurgia aberta e a nefrectomia. Entre esses procedimentos, estão a colocação de um stent ureteral, drenagem percutânea guiada por exame de imagem, nefrostomia, angiografia diagnóstica e angioembolização ${ }^{2}$. No IJF, o serviço de hemodinâmica se encontra em implantação e, como o paciente evoluiu sem complicações durante o acompanhamento, não foi necessário outro tipo de intervenção.

A maioria das lesões renais podem ser acompanhadas com exames laboratoriais e pela avaliação clínica. Os pacientes sintomáticos acabam sendo submetidos a múltiplas tomografias de abdômen, mas a maioria dos pacientes só realiza um exame de imagem contrastado. Os pacientes com lesão renal grau IV e extravasamento urinário de contraste devem ser monitorados com repetição precoce de um exame de imagem para orientar a conduta ${ }^{11}$.

No trauma, a terapia nutricional precoce deve ser iniciada logo que houver estabilidade hemodinâmica, preferencialmente, em até 48 horas da admissão hospitalar ${ }^{15}$. O paciente do caso teve sua dieta iniciada no $3 \mathrm{DIH}$ devido à dúvida sobre a conduta que esteve relacionada à queda da hemoglobina após a primeira transfusão.

Há uma escassez de evidências de alto nível e orientação específica sobre o manejo de pacientes com trauma renal grau IV associado a uma lesão esplênica com grande hemoperitônio. Observou-se que a conduta conservadora pode ser utilizada em um hospital de referência em trauma, usando os mesmos critérios para o tratamento conservador de vísceras maciças. No entanto, existem situações limítrofes que precisam ser melhor compreendidas.

\section{REFERÊNCIAS}

1. Saltzherr TP, van der Vlies $\mathrm{CH}$, van Lienden $\mathrm{KP}$, Beenen $\mathrm{LF}$, Ponsen $\mathrm{KJ}$, van Gulik TM, et al. Improved outcomes in the non-operative management of liver injuries. HPB. 2011May; 13(5):350-5. doi: 10.1111/j.1477-2574.2011.00293.x.

2. Voelzke BB, Leddy L. The epidemiology of renal trauma. Transl Androl Urol. 2014 Jun; 3(2):143-149. doi: 10.3978/j.issn.2223-4683.2014.04.11.

3. Silva LF, Teixeira LC, Rezende JB Neto. Abordagem do trauma renal - artigo de revisão. Rev Col Bras Cir. 2009 Nov-Dez; 36(6):519-24. doi: http://dx.doi. org/10.1590/S0100-69912009000600011.

4. Kautza B, Zuckerbraun B, Peitzman AB. "Management of blunt renal injury: what is new?". Eur J Trauma Emerg Surg. 2015 Jun; 41(3): 251-258. doi: $10.1007 /$ s00068-015-0516-x

5. Dagenais J, Leow JJ, Haider AH, Wang Y, Chung BI, Chang SL, et al. Contemporary Trends in the Management of Renal Trauma in the United States: A National Community Hospital Population-based Analysis. Urology. 2016 Nov; 97: 98-104. doi: 10.1016/j.urology.2016.06.051.

6. Stassen NA, Bhullar I, Cheng JD, Crandall ML, Friese RS, Guillamondegui OD, et al. Selective nonoperative management of blunt splenic injury: an Eastern
Association for the Surgery of Trauma practice management guideline. J Trauma Acute Care Surg. 2012 Nov; 73(5 Suppl 4): 294-300. doi: 10.1097/ TA.0b013e3182702afc.

7. Broska CA Júnior, Linhares AC, Luz AM; Naufel CR Júnior, Oliveira MS, Benção $A L$, et al. Perfil dos pacientes vítimas de trauma renal atendidos em um hospital universitário de Curitiba. Rev. Col. Bras. Cir. 2016; 43 (5): 341-347. doi: 10.1590/0100-69912016005008.

8. Fonseca OCL Neto, Vasconcelos R. Tratamento não cirúrgico do traumatismo renal contuso. JBM. 2013 Nov-Dec; 101(6):35-7.

9. Fernandes TM, Dorigatti AE, Pereira BMT, Cruvinel J Neto, Zago TM, Fraga GP. Tratamento não operatório de lesão esplênica grau IV é seguro usando-se rígido protocolo. Rev. Col. Bras. Cir. 2013 Jul-Ago; 40(4): 323-329. doi: http://dx.doi. org/10.1590/S0100-69912013000400012.

10. Jeremitsky E, Smith RS, Ong AW. Starting the clock: defining nonoperative management of blunt splenic injury by time. Am J Surg. 2013 Mar; 205(3):298 301. doi: 10.1016/j.amjsurg.2012.10.022.

11. Breen KJ, Sweeney P, Nicholson PJ, Kiely EA, O’Brien MF. Adult Blunt Renal 
Trauma: Routine Follow-up Imaging Is Excessive. Urology. 2014 Jul; 84(1): 62-67. doi: 10.1016/j.urology.2014.03.013.

12. Alvarez BD, Razente DM, Lacerda DA, Lother NS, Von-Bahten LC, Stahlschmidt CA. Avaliação do Escore de Trauma Revisado (RTS) em 200 vítimas de trauma com mecanismos diferentes. Rev Col Bras Cir. 2016 Set-Out; 43(5): 334-340. doi: http://dx.doi.org/10.1590/0100-69912016005010.

13. Aragona $F$, Pepe $P$, Patanè $D$, Malfa $P$, D’Arrigo $L$, Pennisi $M$. Management of severe blunt renal trauma in adult patients: a 10-year retrospective review from an emergency hospital. BJU Int. 2012;110 (5):744-748.
14. Dugi DD, Morey AF, Gupta A, Nuss GR, Sheu GL, Pruitt JH. American Association for the Surgery of Trauma grade 4 renal injury substratification into grades 4a (low risk) and 4b (high risk). J Urol. 2010 Feb; 183(2): 592-597. doi: 10.1016/j.juro.2009.10.015.

15. Biscudo-Salomão A, Moura RR, Aguilar Nascimento JE. Terapia nutricional precoce no trauma: após o A, B, C, D, E, a importância do F (FEED). Rev. Col. Bras. Cir. 2013; 40(4): 342-346. doi: http://dx.doi.org/10.1590/S010069912013000400015

\section{Como citar este artigo/How to cite this article:}

Barreira MA, Lima DS, Lopes JAL Filho, Sousa BG, Saldanha ELDD, Sales PRM. Tratamento não operatório de lesão renal e esplênica: relato de caso. J Health Biol Sci. 2018 Out-Dez; 6(4):469-470. 